\title{
THE DETERMINATION OF RESIDUAL STRESS IN EXTRUDED TI-6AL-4V BY CONTOUR METHOD AND FINITE ELEMENT ANALYSIS
}

\author{
J.D.Pollard ${ }^{1}$, S.Rahimi² ${ }^{2}$ A.Watford ${ }^{3}$, M.Jackson ${ }^{1}$, B.P.Wynne ${ }^{1,2}$ \\ ${ }^{1}$ Department of Materials Science and Engineering, The University of Sheffield, Mappin Street, Sheffield S1 3JD, UK \\ ${ }^{2}$ Advanced Forming Research Centre (AFRC), University of Strathclyde, 85 Inchinnan Drive, Inchinnan, Renfrewshire PA4 9LJ, UK \\ ${ }^{3}$ RTI Extrusions Europe, Ltd. (U.K.), Brighouse Road, Low Moor, Bradford, BD12 0QL, U.K.
}

\author{
Keywords: Extrusion, Residual Stress, Ti-6Al-4V, FEM, Contour Method
}

\begin{abstract}
The contour method has been used to assess the level of residual stress generated during the post extrusion air cool and water quenching of a Ti-6Al-4V extruded bar. A length of the extrudate was transversally sectioned using electrical discharge machining (EDM), resulting in minimal damage to the cut surface; the stress relieved profile present at the cut surface was then measured using a coordinate measuring machine (CMM). The cut section was recreated in an ABAQUS finite element (FE) model and the inverse of the stress relieved profile recorded by CMM was applied as a boundary condition; by forcing the model's surface topology to match the measured cut surface, residual stresses normal, i.e. longitudinal stresses, to the cut surface of the extruded profile were then calculated. Results were compared to air cooling and quenching simulations performed in the DEFORM FE package. The distribution of tensile and compressive residual stresses observed via experimental and simulation work were found to be a good match with the distribution correlating with local variations of cooling rate during quenching.
\end{abstract}

\section{Introduction}

Extruded Ti-6Al-4V sections are used in the aerospace sector as a means of reducing costs by providing near net shape parts that require limited machining to produce the final component. The sections are extruded at high temperature; post-extrusion the sections are then often quenched to room temperature. This processing route introduces residual stresses into the extrudate that can result in distortion of the profile of the extruded bar post quench or when it is machined to produce the final component. While the full processing route often involves annealing stages after the extrusion to ameliorate some of these problems, this work focuses on determining residual stresses present in the asquenched state, using both experiments and numerical modelling, as a means of understanding the key variables that determine their presence.

Residual stresses can be present in a component as a result of both its thermal history and its deformation history [1,2]. During mechanical processing residual stresses are produced as a result of strain accommodation that arises as a result of inhomogeneous plastic deformation across a workpiece, where plastic flow is localised in certain regions of the workpiece [1]. Similarly nonuniform expansion and contraction of the workpiece during thermal processing will introduce residual stresses via the same strain accommodation mechanism [1]. It has also been suggested that strain-rate will also have an effect on the residual stresses generated in a component [3].
Discussions of residual stresses in extruded materials are typically in the context of cold extrusion. Die angle, reduction in area and the factors related to the deformation zone are held to be significant factors in the distribution of residual stress; for rapid extrusions the temperature gradient due to inhomogeneous deformation and the subsequent cooling may also contribute [4]. The extruded Ti-6Al-4V component investigated in this work was subjected to extreme strains, strain rates and significant thermal gradients during its manufacture. The mentioned processing parameters may be responsible for introducing some residual stresses into the component; however, it is thought that for this particular processing route the very high processing temperature will reduce the impact of inhomogeneous deformation and high strain-rates as stress introduction factors. Non-uniform cooling rates of the extrudate post-extrusion are considered as the most significant source of residual stress for these components.

There are a number of techniques that can be used to determine the residual stresses present within a body including hole drilling, ultrasonic methods, synchrotron methods and x-ray and neutron diffraction $[5,6]$. Different methods have benefits and drawbacks, in this instance the contour method is used as it is a high resolution technique that is suitable for measuring full $2 \mathrm{D}$ residual stress map in large components [5]. In this technique the displacements resulting from stress-relief at a cut surface are mapped and then used as a boundary condition on a flat surface in a finite element (FE) model using superimposition principal, leading to a direct calculation of residual stress [7,8]. There are numerous instances of the contour method being used to successfully assess residual stresses present in Ti-6Al-4V components that have been subjected to a range of processing methods and conditions [9-12].

\section{Methodology}

The extruded bar used in this work was a $500 \mathrm{~mm}$ long section of extruded Ti-6Al-4V with a complex cross-section, schematically shown in figure 1 . The dimensions of the as-received bar were measured using a Nikon Evolution Coordinate Measurement Machine (CMM) located at the Advanced Manufacturing Research Centre, the University of Sheffield. A series of 10 points along the length of the bar were measured at nine points around the profile, five of these locations (shown in figure 1) were then used to assess the flatness of the bar.

The bar was then heated to $1100^{\circ} \mathrm{C}$, i.e. super beta transus, in a furnace for 1 hour before cooling in air for 60 seconds followed by quenching in a tank of room temperature water (shown schematically in figure 2). Note, the bar was carefully lowered into the quench tank with the smaller flange section first by means of two wires attached to the ends of the bar through the thicker 
flange section. The distortion of the as-quenched bar was then measured at the same points using the same procedure as previously described. The CMM measurements used to measure the flatness of the bar were processed in Matlab and plotted against nominal measurements, as given by technical drawings of the extrudate.

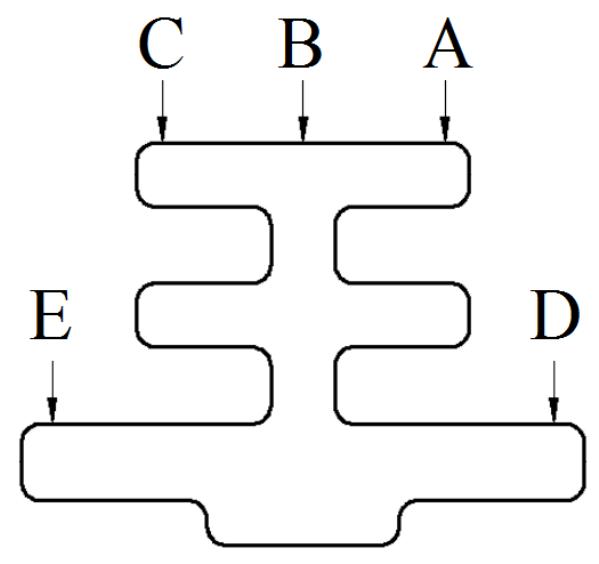

Figure 1. A schematic of the extruded profile with CMM measurement locations marked (Not to scale).

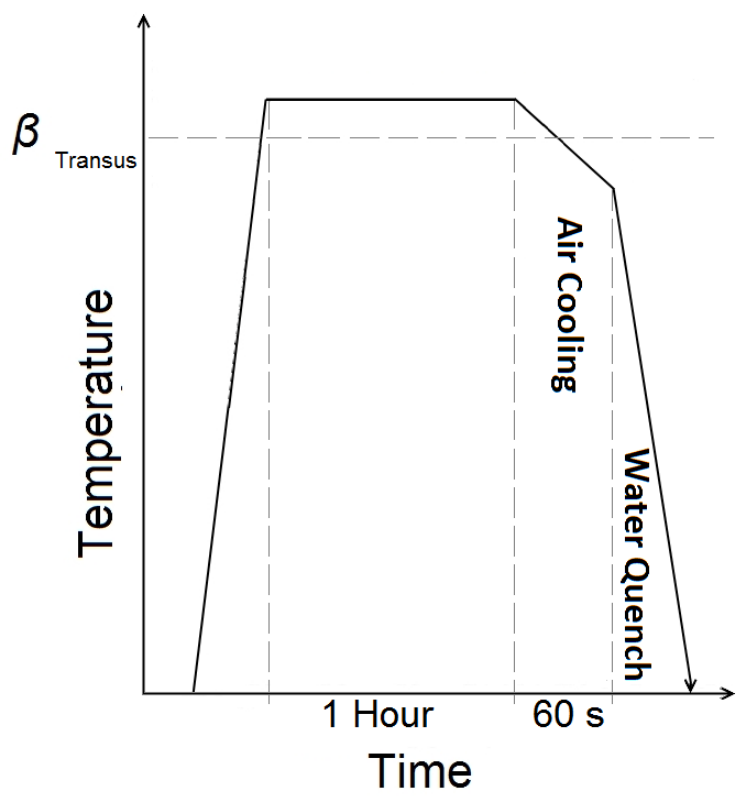

Figure 2. A schematic diagram of the heat treatment and cooling carried out in the experiment.

To generate the stress relieved profile surfaces for the contour method the bar was clamped at both sides and sectioned at its mid-point by means of an electrical discharge machine (EDM), in skim cut mode, equipped with a $150 \mu \mathrm{m}$ zinc coated brass wire. Sacrificial plates were also machined with the same profile as the bar and bonded to it using conductive epoxy in order to create a uniform cross-section (an example is shown in figure 3) to allow accurate measurements up to the edges of the profile shape. The surface distortion at the cut surfaces was then measured in $0.1 \mathrm{~mm}$ steps using a Mitutoyo Crystal Apex C CMM equipped with a Renishaw PH10T probe with a $1 \mathrm{~mm}$ diameter ruby tip located at the Advanced Forming Research Centre, the University of Strathclyde.

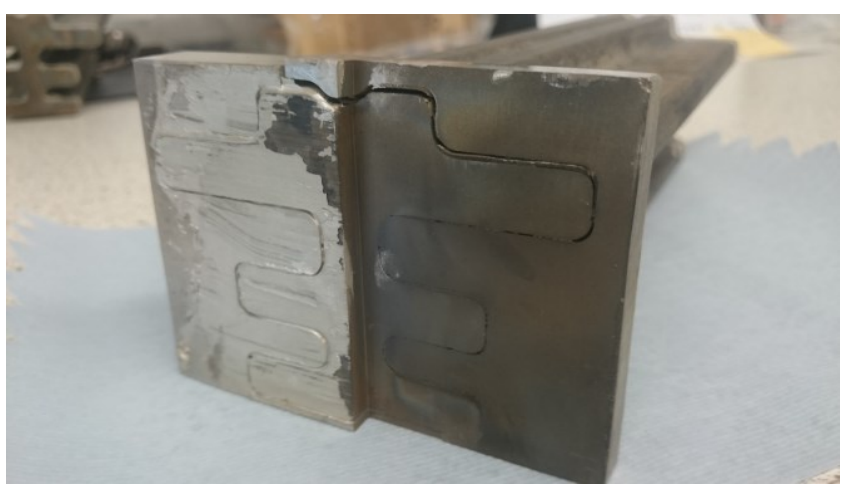

Figure 3. The bar after cutting, the sacrificial plates can clearly be seen in this image.

The data recorded by the CMM measurements of the stress relieved profiles were post processed to ensure measurements from both faces of the cut were aligned and any outlying points and flat plane data were removed. The processed data from both surfaces of the cut were then averaged and used to construct a single dataset to which a series of bivariate splines were fitted. Selection of the spline that best fitted the CMM dataset was achieved by analysing a plot of the residuals of the entire 2D surface to determine which spline most closely matched the data points

The un-deformed geometry of half of the extruded bar was created by extruding the section profile to a length of $250 \mathrm{~mm}$ in ABAQUS; the geometry was meshed using a $0.5 \mathrm{~mm}$ element size on the cut surface. The element size was biased to $20 \mathrm{~mm}$ toward the end of the extrudate. The inverse spline surface generated from the CMM measurements was applied to the FE model as a boundary condition to reproduce the surface relieved profile of the physical specimen; the stresses at the deformed surface were then extracted for analysis. A Young's Modulus of $113.8 \mathrm{GPa}$ and a Poisson's ratio of 0.325 were used as material inputs for the model [13].

FE simulations of the full cooling profile of the bar were performed in the FE package DEFORM using quarter symmetry models in order to compare the trends from the CMM flatness measurements and the distribution of residual stresses at the mid cross-section of the extruded bar. The FE model was constructed using $0.5 \mathrm{~mm}$ sized tetrahedral elements resulting in a mesh comprised of approximately 700,000 elements.

Heat transfer coefficients (HTCs) for the simulation work were determined from inverse heat analysis of air cooling and quenching trials carried out using an extruded section of Ti-6Al$4 \mathrm{~V}$ of different profile to that shown in figure 1 . The cross-section geometry and overall dimensions of this profile were sufficiently similar to the one shown in figure 1 that the HTCs calculated were considered a suitable approximation for use in the current simulations. These trials consisted of heating the section of bar to $1100^{\circ} \mathrm{C}$ in a chamber furnace before first cooling in air and then water quenching it in a subsequent experiment. During cooling the 
temperatures around the profile were measured using type $\mathrm{N}$ thermocouples inserted near the surface of the section; data was recorded using a NI 9213 thermocouple interface with a NI cDAQ-9171 USB chassis. The cooling curves recorded were processed in DEFORM's inverse heat analysis module and HTCs were calculated as a function of temperature. For the $60 \mathrm{~s}$ period of time where the section cools in air the HTCs ranged from 0.47 $\mathrm{N} / \mathrm{s} / \mathrm{mm} /{ }^{\circ} \mathrm{C}$ at $1100^{\circ} \mathrm{C}$ to $0.036 \mathrm{~N} / \mathrm{s} / \mathrm{mm} /{ }^{\circ} \mathrm{C}$ at $800^{\circ} \mathrm{C}$. The HTCs used for the quench part of the simulations varied between 7.5 $\mathrm{N} / \mathrm{s} / \mathrm{mm} /{ }^{\circ} \mathrm{C}$ and $0.28 \mathrm{~N} / \mathrm{s} / \mathrm{mm} /{ }^{\circ} \mathrm{C}$; the values of these HTCs reflect the reduced heat transfer effects seen in the cooling curves that occur as a result of film and nucleate boiling that occur during water quenching. Consequently, the HTCs at higher temperature are lower than some of the HTCs at lower temperatures due to boiling at the interface between the specimen and the quench medium producing an insulating gas layer that diminishes as the temperature decreases.

The coefficient of thermal expansion (CTE) values for Ti-6Al-4V are a weighted average of the CTEs of the $\alpha$ and $\beta$ phases respectively [14]. The CTE values used for the $\mathrm{FE}$ simulations were between $9.0 \times 10^{-6} /{ }^{\circ} \mathrm{C}$ at room temperature to $11.7 \times 10^{-6} /{ }^{\circ} \mathrm{C}$ beyond the transus temperature of $1001^{\circ} \mathrm{C}$, though other values are reported elsewhere, with alternative $\mathrm{CTEs}$ values ranging from $9.5 \times 10^{-6} /{ }^{\circ} \mathrm{C}$ up to $12.8 \times 10^{-6} /{ }^{\circ} \mathrm{C}$ during the $\alpha+\beta$ to $\beta$ transformation [14-16].

The simulation work conducted aimed to model the residual stresses and the associated distortion produced as a result of variation in the thermal expansion of the material with temperature. In reality the variation in volume fraction of the $\alpha$ and $\beta$ phases with temperature, their respective capacity to locally absorb strains and stresses as the material transforms from $\beta$ to $\alpha+\beta$ at $1001^{\circ} \mathrm{C}$ will also affect the residual stresses generated in the bar. In this work the impact of phase transformation is not accounted for in the simulations and, as such, will have an effect on the accuracy of simulation residual stress results compared to those recorded experimentally.

\section{Results}

The results of flatness measurements are shown in figure 4 and figure 5; nominal flatness of the locations where CMM measurements were taken is based on original technical drawings and is represented by flat planes.

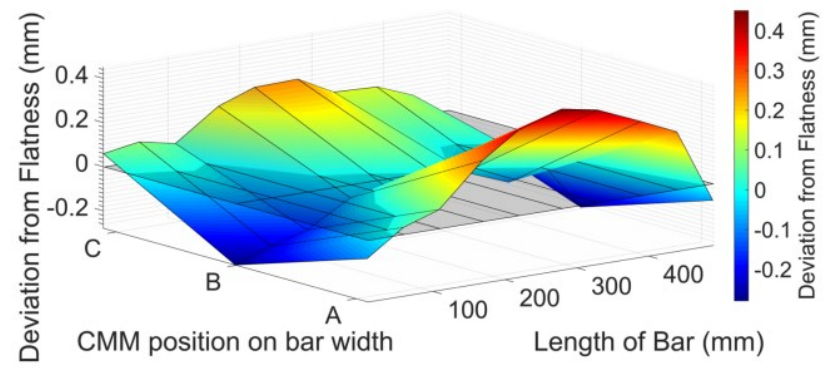

Figure 4. A comparison of the flatness of the upper surface of the quenched bar with nominal dimensions.

CMM measurements taken before the quench experiment were used to measure the extent of the deformation produced by the quenching process compared to the as-received bar; figure 6 and figure 7 show the post-quench flatness measurements after accounting for the deviations from the nominal tolerances present in the pre-quenched bar, for the top and bottom surfaces respectively. It should be noted, however, in the as-received condition the bar had not been subjected to the full extrusion product processing window, where annealing and straightening steps are performed.

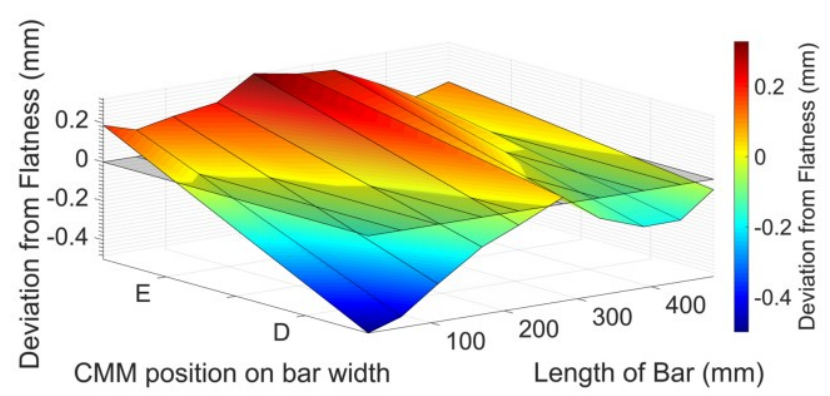

Figure 5. A comparison of the flatness of the lower surface of the quenched bar with nominal dimensions.

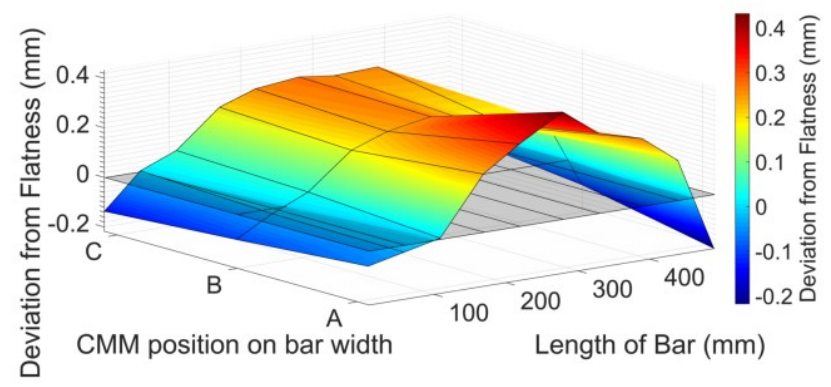

Figure 6. The post quench deformation after compensating for initial deviations from nominal tolerances.

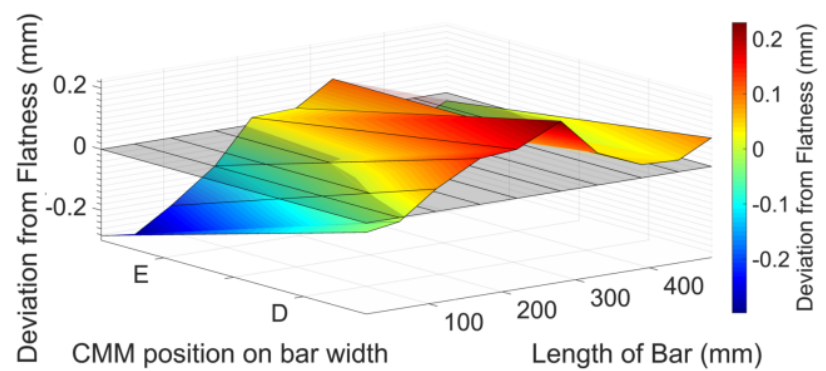

Figure 7. The lower surface CMM measurements after accounting for pre-quench deviations from nominal tolerances

As previously described, the displacement map of the EDM cut surface measured by CMM was used as boundary condition for an FE simulation to match the displacement map. The normalized stresses produced at the cut surface of the FE model by this procedure are given in figure 8 , where positive numbers indicate tensile stresses and negative numbers compressive stresses.

Figure 9 and figure 10 show the distortion of the top surface predicted by DEFORM at the end of the air cooling and water 
quenching periods respectively; the lower surface reflected the same trends.

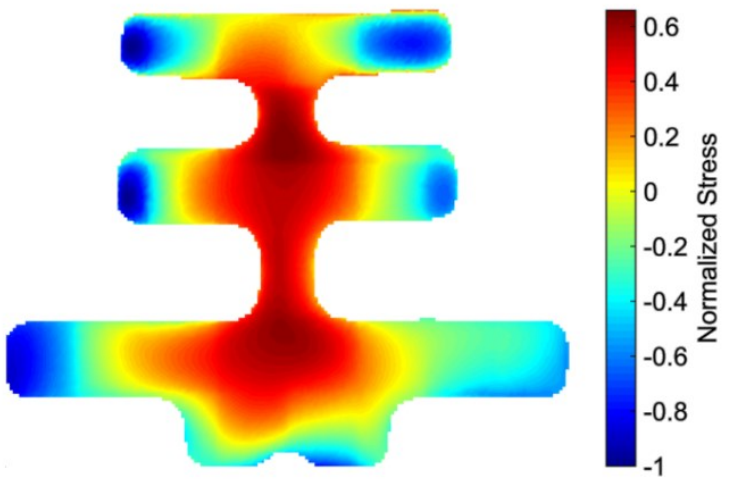

Figure 8. The surface stress measured experimentally from averaged data points.

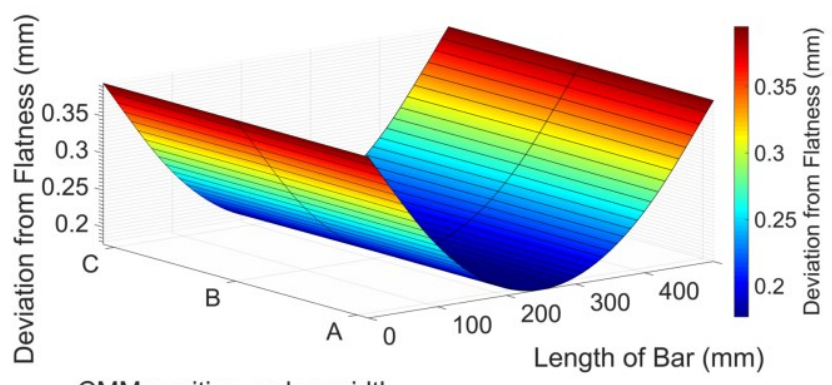

CMM position on bar width

Figure 9. Curvature of the bar's top surface after $60 \mathrm{~s}$ in air as predicted by FE simulation.

The normalized residual stresses predicated purely from FE modelling of the cooling process are shown in figure 11 .

\section{Discussion}

A key assumption of the work presented here is that any residual stresses and/or distortion of the bar measured is produced solely as a result of the air cool and water quench undertaken and the associated phase changes during cooling. The temperature soak of 1 hour at $1100^{\circ} \mathrm{C}$ should have provided sufficient conditions for the stress-relaxation of any residual stresses introduced into the section during the prior extrusion and cooling processes.

The CMM measurements of the flatness of the bar after aircooling and quenching indicate a clear curvature of the bar along its top surface in the extrusion direction. The extent of the deviations from nominal dimensions due to this curvature are small along the $500 \mathrm{~mm}$ length with the largest deflection being under $0.5 \mathrm{~mm}$. In the scope of the $500 \mathrm{~mm}$ length of section deviations of less than $0.5 \mathrm{~mm}$ fall well within the permissible tolerances for the component, however, across the length of a full extrusion of length 6 to $8 \mathrm{~m}$ it is likely that the curvature measured would result in the parts of the bar at either extreme of the length being outside of acceptable tolerance limits.

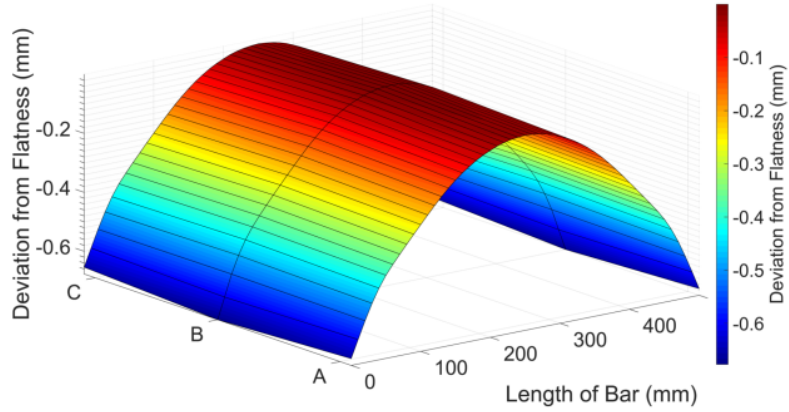

CMM position on bar width

Figure 10. Curvature of the bar's top surface after quenching as predicted by FE simulation.

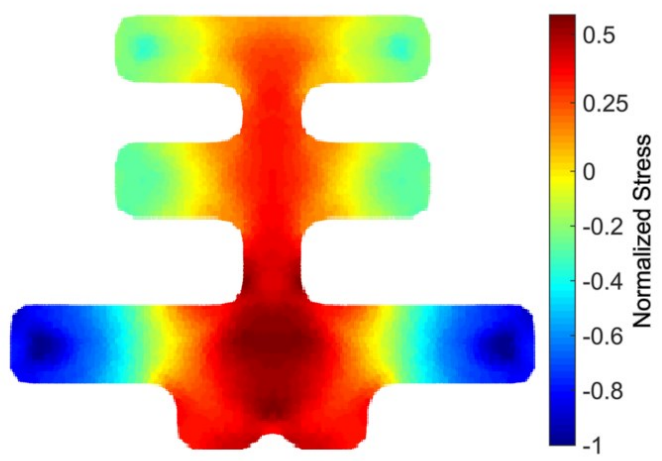

Figure 11. The residual stress distribution predicted by FE modelling.

When examining the distribution of the measured residual stresses around the profile (figure 8) a correlation can be made between the regions of compressive and tensile residual stresses and the distortions recorded by CMM measurement, (figure 4 and figure 5). The regions where high compressive stresses were recorded in figure $8,(\mathrm{CMM}$ measurement locations $\mathrm{A}, \mathrm{C}$ and $\mathrm{E})$ correspond to the regions showing the greatest distortion from nominal dimensions. This is particularly evident at the outer edges of the top surface (locations $\mathrm{C}$ and $\mathrm{A}$ in figure 1); at the centre of the bar these two points have some of the largest displacements away from the tolerance, and per figure 8 it is clear that they are subjected to the largest compressive stresses recorded in the profile. Similarly, comparison of the distortion of the lower surface of the profile indicates that at the centre of the bar the measurements taken from the E set of CMM data correspond to another significant deviation from nominal dimensions which again correspond to a region of high compressive stress in figure 8. Compressive residual stresses of a lower magnitude were recorded in the location of the CMM measurements for the dataset $\mathrm{D}$ at the centre of the bar; accordingly the CMM measurements show a smaller deviation from nominal dimensions. Finally, it can be seen from figure 4 that for the CMM dataset $\mathrm{B}$, the point at the centre of the top surface of the bar shows minimal difference to the nominal dimensions; examining the distribution of residual stresses this corresponds to a region of low tensile stress. 
As previously stated, it has been assumed that the distortion observed in the post-quench bar is purely the result of the heat treatment work carried out. In the as-received condition the bar exhibited some distortion relative to the nominal tolerances; to accommodate the possibility that some of the dimensions recorded in figure 4 and figure 5 incorporate some prior difference from nominal dimensions, despite stress-relieving the bar during the heat treatment, the stress distribution recorded was compared to measurements that assume distortion recorded prior to the heat treatment was retained. These measurements, shown in figure 6 and figure 7, still show a good match between the regions with the greatest distribution and the recorded residual stress distributions.

Quench simulations of the extruded bar match the flatness trend presented from CMM measurements of the extruded and quenched bar. CMM measurements indicate the bar develops a curvature along its length during cooling as shown in figure 4 . Simulations followed the same process as the experiment where 60 seconds of air cooling was followed by a water quench; unlike the experiment, simulations allowed the deformation of the bar to be predicted throughout the process. During the air cooling portion of the simulation, measurements indicated that the bar developed a curvature opposite to the curvature direction recorded by the CMM flatness measurement as shown in figure 9. The bar in the simulation then bends in the opposite direction during the water quenching section of the simulation to match the curvature trend recorded by the CMM measurements (figure 10). While the simulations match the general shape distortion along the length of the bar the upward movement of the flanges at locations $\mathrm{A}$ and $\mathrm{C}$ are not accurately predicted by FE, this may be an effect of local variations in the HTCs and suggests that accuracy of the model may be improved by further tailoring the HTCs used in the simulations to better account for local variations in heat transfer across the section profile.

The distribution of residual stress around the profile corresponds with the variation in expected cooling rates at different sections of the profile geometry. The tips of the thinner sections of the profile where the highest cooling rates are predicted show the highest values of residual compressive stress; the thicker section where the lowest cooling rates are experienced has some of the highest measured values of tensile stress in the specimen. Of interest is the fact that the central vertical section of the profile also contained high levels of tensile residual stresses; the thinness of this part of the section would lead to expectations of relatively high cooling rates. The presence of tensile stresses suggests that this region cools more slowly than predicted; one possible explanation for a reduced cooling rate in these regions during quenching is a lower heat transfer from the surface due to film and nucleate boiling producing trapped pockets of gas in recessed parts of the geometry and the lower surface.

While the FE simulation does not model effects such as film and nucleate boiling during the water quench, the cooling curves produced during the prior experimental work reflect the reduced heat transfer that is a consequence of these effects. Hence, the HTCs determined by inverse heat analysis of this data effectively account for the reduced heat transfer experienced by the component during parts of the quench and low cooling rates are observed in the central region of the profile during the simulation. The ratio of the magnitudes of the compressive and tensile stresses between the experimental and simulation results are a close match. The stress distribution produced by the FE simulation generally mirrors that which was determined by the contour methods though the compressive stresses recorded at the ends of the shorter flanges (locations $\mathrm{A}$ and $\mathrm{C}$ and their counterparts in the middle flange section) are of a lower magnitude. Similarly the distribution of tensile stresses in the FE simulation is also a reasonable match to the experimental work; however, the tensile stresses in the FE simulation extend out to the bottom edges of the profile where compressive stress is seen in the actual section. While the HTCs applied in the simulation clearly allow a reasonable prediction of the stress distribution in the section assessed, they were not produced using the same geometry and hence this could account for the variances observed between the simulation and experimental results. Additional variations may due to factors not accounted for in the simulation material model including transformation induced stresses and crystal plasticity.

It is important to note that the orientation of the section during the quench could have a significant effect on the stress distribution produced during quenching from high temperature. If the section were rotated through $90^{\circ}$ relative to its orientation in figure 1 and then quenched from temperature, the geometry would produce pockets of gas that were trapped in the recesses on the lower side resulting in lower cooling rates while the recesses on the upper side would not trap any gas and consequently would cool more rapidly. This asymmetric cooling would produce a similarly asymmetric residual stress distribution that could cause significant problems when the extruded component is machined down to the final component tolerances.

\section{Conclusion}

The contour method for measuring residual stress has been successfully applied to measure the as-quenched longitudinal residual stresses in a Ti-6Al-4V extrusion with a complicated cross-section. This has led to the following conclusions:

- FE simulations show comparable residual stress results, even without taking phase transformation into account, to those obtained via the contour method. Consequently, FE simulations have much potential to be used to predict residual stress distributions in extruded components and thus be used to optimise the processing route to reduce them, where possible.

- Residual stresses in Ti-6Al-4V components that are extruded at super beta transus temperatures will be distributed around the profile location according to the cooling rates experienced at different parts of the profile, which in turn are dependent on the geometry of the profile, particularly thickness.

- Quenching orientation of an extruded component may have a measurable effect on residual stress distribution as non-symmetric profiles with recesses may produce pockets of trapped gas where lower cooling rates occur during quenching.

\section{Acknowledgements}

J.D.Pollard is part of the ESPRC Centre for Doctoral Training in Advanced Metallic System and gratefully acknowledges the financial support of the Engineering and Physical Sciences Research Council, UK. Thank you also to the technical staff at the University of Sheffield for their assistance with the high 
temperature quenching work and to $\mathrm{Mr}$ Ioannis Violatos for discussions on the optimization of FE quench simulations.

\section{References}

1. George E. Dieter, Mechanical Metallurgy (McGraw-Hill, 1988), 557-558.

2. P.J. Withers, "Residual stress and its role in failure," Reports Prog. Phys. 70 (2007), 2211-2264.

3. A.K. Tiwari, A.R. Patel and N. Kumar, "Investigation of strain rate on residual stress distribution," Mater. Des. 65 (2015), 1041-1047.

4. Z. Wang and B. Gong, B., "Residual Stress in the Forming of Materials," in ASM Handbook of Residual Stress and Deformation of Steel (G.E. Totten, ed.), (ASM International, 2002), 141-147.

5. N.S. Rossini, M. Dassisti, K.Y. Benyounis and A.G. Olabi, "Methods of measuring residual stresses in components," Mater. Des. 35 (2012), 572-588.

6. F.A. Kandil, J.D. Lord, A.T. Fry and P.V.A. Grant, "A review of residual stress measurement methods - A guide to technical selection" (NPL Report MATC(A)04, National Physical Laboratory, 2001).

7. M.B. Prime, "Cross-Sectional Mapping of Residual Stresses by Measuring the Surface Contour After a Cut," J. Eng. Mater. Technol. 123, 162-168 (2001).

8. F. Hosseinzadeh, J. Kowal and P.J. Bouchard, "Towards good practice guidelines for the contour method of residual stress measurement," J. Eng. (2014), doi:10.1049/joe.2014.0134

9. P. Xie, H. Zhao, W. Bing, and S-L. Gong, "Using Finite Element and Contour Method to Evaluate Residual Stress in Thick Ti-6Al-4V Alloy Welded by Electron Beam Welding," Acta Metall. Sin. (Engl. Lett.) (2015), doi:10.1007/s40195-015-0276-y

10. K.K. Liu and M.R. Hill, "The effects of laser peening and shot peening on fretting fatigue in Ti-6Al-4V coupons," Tribol. Int. 42 (2009), 1250-1262.

11. P. Frankel, M. Preuss, A. Steuwer, P.J. Withers and S. Bray, "Comparison of residual stresses in Ti-6Al-4V and Ti-6Al-2Sn4Zr-2Mo linear friction welds," Mater. Sci. Technol. 25 (2009), 640-650.

12. S.D. Cuellar, M.R. Hill, A.T. Dewald and J.E. Rankin, "Residual stress and fatigue life in laser shock peened open hole samples,” Int. J. Fatigue 44 (2012), 8-13.

13. ASM/Aerospace Specification Metals Inc. Titanium Ti-6Al4V Grade 5 (Annealed) (2015).

14. J.W. Elmer, T.A. Palmer, S.S. Babu, and E.D. Specht, "Low temperature relaxation of residual stress in Ti-6Al-4V," Scr. Mater. 52 (2005), 1051-1056.
15. RMI Titanium Company. Titanium alloy guide. (2000).

16. A.K. Swarnakar, O. Van Der Biest and B. Baufeld, "Thermal expansion and lattice parameters of shaped metal deposited Ti6Al-4V," J. Alloys Compd. 509 (2011), 2723-2728. 